\title{
Morbidity due to Schistosoma mansoni: an epidemiological assessment of distended abdomen syndrome in Ugandan school children with observations before and 1-year after anthelminthic chemotherapy
}

\author{
Julie Balen a,b,c, J. Russell Stotharda,d,*, Narcis B. Kabatereine ${ }^{e}$, \\ Edridah M. Tukahebwa ${ }^{e}$, Francis Kazibwe ${ }^{e}$, Sarah Whawell ${ }^{a}$, \\ Joanne P. Webster ${ }^{a}$, Jürg Utzinger ${ }^{b}$, Alan Fenwick ${ }^{a}$ \\ a Department of Infectious Disease Epidemiology, Faculty of Medicine, Imperial College London, London, UK \\ b Department of Public Health and Epidemiology, Swiss Tropical Institute, Basel, Switzerland \\ c Department of Population Health, Queensland Institute of Medical Research, University of Queensland, Brisbane, Australia \\ ' Department of Zoology, Natural History Museum, London, UK \\ e Vector Control Division, Ministry of Health, Kampala, Uganda
}

Received 14 October 2005; received in revised form 8 December 2005; accepted 8 December 2005

Available online 12 June 2006

\section{KEYWORDS}

Intestinal

schistosomiasis;

Schistosoma mansoni;

Morbidity;

Hepatosplenomegaly;

Ascites;

Deworming;

Uganda

\begin{abstract}
Summary The objectives of this study were to determine the prevalence and distribution of distended abdomens among Ugandan school children across a range of eco-epidemiological settings and to investigate the relationship between distended abdomens and helminth infections, in particular Schistosoma mansoni, before and 1-year after anthelminthic treatment. A cross-sectional survey was conducted on 4354 school children across eight districts, with a longitudinal 1-year follow-up of 2644 children $(60.7 \%)$. On both occasions, parasitological, biometrical and clinical data were collected for each child. Baseline prevalence of S. mansoni and hookworms was $44.3 \%$ and $51.8 \%$, respectively. Distended abdomens, defined as an abdominal circumference ratio (ACR) $>1.05$, were observed in $2.5 \%$ of the sampled children, several of whom presented with particularly severe distensions necessitating hospital referral. ACR scores were highly overdispersed between districts and schools. Multivariate regression analysis revealed that $S$. mansoni infection accounted for only a small fraction of ACR variation, suggesting that either single point prevalence and intensity measures failed to reflect this more chronically evolved morbidity and/or that other interacting factors were involved,
\end{abstract}

\footnotetext{
* Corresponding author. Tel.: +44 207942 5490; fax: +44 2079425518.

E-mail address: r.stothard@nhm.ac.uk (J.R. Stothard).
} 
e.g. malnutrition and malaria. At 1-year follow-up, ACR scores showed an overall trend of regression towards the mean, potentially indicative of amelioration following chemotherapy, but geographic overdispersion still remained.

(c) 2006 Royal Society of Tropical Medicine and Hygiene. Published by Elsevier Ltd. All rights reserved.

\section{Introduction}

Schistosomiasis is a chronic, debilitating, poverty-related disease, and in many parts of sub-Saharan Africa it continues to drain the socio-economic development of already impoverished rural communities (Engels et al., 2002; King et al., 2005; Raso et al., 2005). Since control of schistosomiasis in high burden areas is primarily concerned with morbidity reduction through the use of regular anthelminthic chemotherapy (Fenwick et al., 2003; Utzinger and Keiser, 2004; WHO, 2002, 2004), interest has increased in better describing the public health burdens of schistosomiasis both at national and local levels (van der Werf et al., 2002, 2003), as well as clearly demonstrating morbidity reversion post treatment (Boisier et al., 1998; Brooker et al., 2004; Lambertucci et al., 2000).

In contrast to urinary schistosomiasis where visual signs of morbidity are often apparent, such as gross haematuria, morbidity associated with intestinal schistosomiasis is often less obvious in all but the most severe or chronic cases (Kongs et al., 2001). Whilst clinical investigations with ultrasound and/or abdominal palpation methods can better describe morbidity associated with Schistosoma mansoni infection, for example hepatosplenomegaly, liver fibrosis and/or organ texture (Hatz, 2001; King et al., 2003), these require specialist equipment and well trained staff (Nooman et al., 1995; Ruiz et al., 2002). As a consequence, large-scale community screening of morbidity has been limited and broad geographic comparisons are rarely made.

In Uganda, intestinal schistosomiasis is present in 38 of 56 districts and poses a considerable public health burden (Kabatereine et al., 1996, 2004a, 2004b; Williams et al., 1986). To reduce the morbidity associated with this disease, a national schistosomiasis and intestinal worm control programme was launched at Pakwach, Nebbi district, in March 2003. The programme aims to provide regular combination anthelminthic treatments to school-age children and communities at high risk of infection and morbidity within the most severely afflicted Ugandan districts (Kabatereine et al., 2004a, 2006). Morbidity associated with intestinal schistosomiasis can be very debilitating, and Ongom and Bradley (1972) recognised the local severity of the disease long ago. The well known visual sign of a grossly distended abdomen, which was frequently seen in adolescent boys (Ongom et al., 1972), is still recognised today by the local community to be an incapacitating disease state, although a precise understanding of its water-borne parasitic aetiology may be lacking (Kabatereine et al., 2004b).

Whilst the visual sign of a distended or swollen abdomen can be clearly associated with advanced morbidity, to our knowledge the occurrence, distribution and severity of this condition have not been investigated within the schoolage community where it likely develops. We therefore determined the prevalence and distribution of distended abdomens among school children across a range of ecoepidemiological settings in Uganda, in order to determine whether a relationship could be found with S. mansoni infection before and 1-year after anthelminthic chemotherapy.

\section{Materials and methods}

\subsection{Study area and population}

A cross-sectional survey at intervention baseline between October 2002 and June 2003 was carried out in 37 primary schools from eight districts in the northwest and southeast of Uganda (Figure 1). Within each district, five sentinel schools were selected from available parasitological mapping data (Kabatereine et al., 2004a) that conformed to a parasitological stratification of S. mansoni as follows: two high prevalence $(\geq 50 \%)$, two medium prevalence $(<50 \%$ and $>10 \%$ ) and one low prevalence $(\leq 10 \%)$ school. It was intended for the latter low prevalence school for each district to act

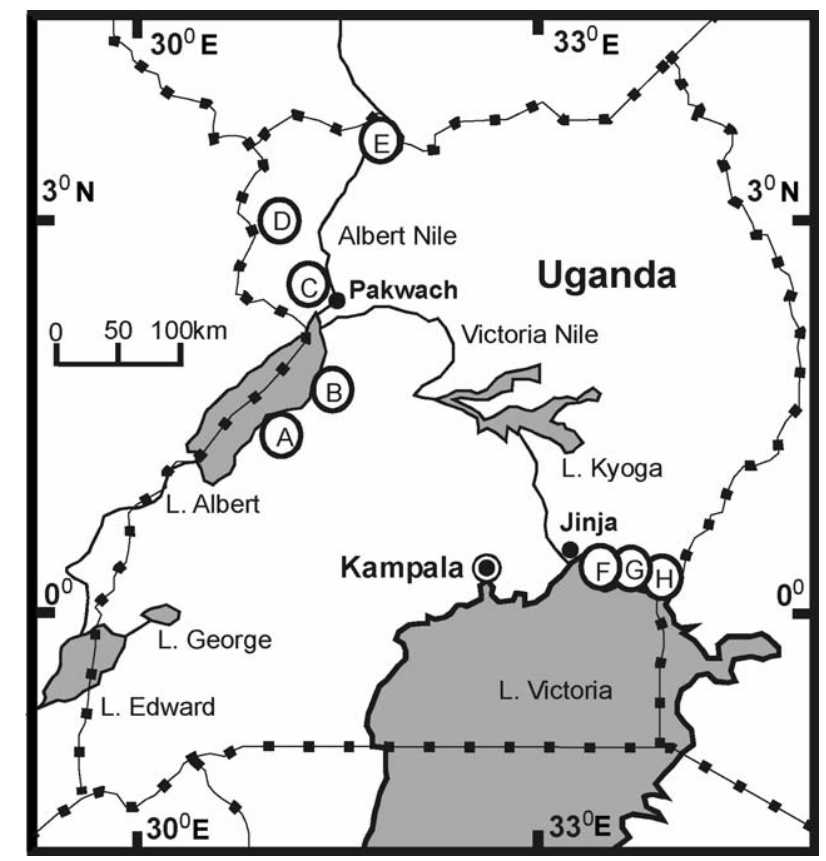

Figure 1 Sketch map of the study areas in Uganda. In total, 37 primary schools from eight districts were visited, including both Lake Albert/West Nile and Victoria environments. $n$ : number of schools examined; A: Hoima $(n=5)$; B: Masindi $(n=5)$; C: Nebbi $(n=5)$; D: Arua $(n=1)$; E: Moyo $(n=6) ; \mathrm{F}$ : Mayuge $(n=5)$; G: Bugiri $(n=5)$; H: Busia $(n=5)$. Note that Panyagoro, where the study cohort of Ongom and Bradley (1972) was located, is found some $5 \mathrm{~km}$ east of Pakwach. 
as a quasi-control enabling better assessment of clinical and biometric scores for each child in the general local absence of intestinal schistosomiasis. Owing to logistic constraints, only a single school was surveyed in Arua district, and in search of additional high prevalence schools six schools were surveyed in Moyo district. For ethical reasons, it was not appropriate to include any untreated control groups in the study design.

Within each school, children were selected randomly on the basis of age to form four cohorts of approximately 15 boys and 15 girls of the following ages: 6, 7, 8 and 11 years, giving a total of approximately 120 per school. Owing to an uncharacteristic age distribution of children attending Tonya primary school, Hoima district, this age cohort structure could not be filled and 80 children were therefore chosen randomly from those available, aged between 6 years and 11 years.

\subsection{Measuring a distended abdomen: the abdominal circumference ratio (ACR)}

Each child's height and weight was measured with a clinical stadiometer and digital solar scales, respectively, the latter of which was re-calibrated daily. To standardise the measurements across children of varying heights for differing levels of abdominal distension, two physical landmarks were chosen to anchor measurements: (i) the xiphoid process of the sternum; and (ii) the umbilicus of the abdomen. Measurements were then taken with dressmakers tape while the child remained standing. Special care was taken that the child was relaxed and fully compliant during measurement. Measurements were taken upon relaxed state after the final expiration of three prior ventilation cycles. The circumference of the thorax around the sternum was measured parallel with the ground beginning and ending on the xiphoid process, whilst the abdominal circumference was measured parallel with the ground beginning and ending on the umbilicus, both to the nearest $5 \mathrm{~mm}$. If the measurements were unsatisfactory, e.g. the child failed to relax, measurements were immediately re-taken. The ACR was then calculated as the umbilical circumference divided by the sternal circumference. The greater the abdominal distension the greater the increase of ACR from unity (i.e. $\geq 1.0$ ). To avoid observation bias, all measurements were taken by the same technician from the Vector Control Division (Ministry of Health, Kampala, Uganda) throughout the study and the technician's performance was regularly crosschecked by one of the authors (J.R.S.).

\subsection{Parasitological diagnosis and further clinical measurements}

Each child provided a single stool sample that was examined for the presence of eggs of S. mansoni and soil-transmitted helminths (i.e. Ascaris lumbricoides, Trichuris trichiura and hookworms) using standard Kato-Katz thick films $(41.7 \mathrm{mg}$ ) according to Katz et al. (1972). Egg counts were recorded for each parasite separately and converted to the number of eggs per gram of faeces (EPG). Watery stools (diarrhoea) were excluded from the analysis owing to their dilutive influence on egg counts. For each school and district, the geometric mean was calculated for S. mansoni egg counts (SMEPG) and hookworm egg counts (HWEPG).

Fingerprick blood samples were taken to assess haemoglobin levels using a B-haemoglobin HemoCue photometer (HemoCue, Ängelholm, Sweden). Further physical examinations were performed on each child by a community nurse who assessed the size and texture of the liver and spleen by palpation. In addition, children from Hoima and Mayuge districts were examined by ultrasound. Protocols and results of abdominal palpation and ultrasound will be reported fully elsewhere (N.B. Kabatereine et al., unpublished data) and hence only salient findings upon comparison with ACR scores are reported here.

\subsection{Anthelminthic treatment and ethical approval}

Regardless of their infection status, all children examined in the surveys were treated with oral combination anthelminthic chemotherapy in conjunction with a food item and soft drink under the supervision of a community nurse. An appropriate medication of $40 \mathrm{mg} / \mathrm{kg}$ praziquantel for S. mansoni, as calculated by child's height according to the amended WHO height pole (Montresor et al., 2005), and a single $400 \mathrm{mg}$ albendazole tablet for intestinal helminths were administered to each child. Children were monitored after treatment for any immediate adverse reactions. Children were also given treatment cards to aid with their identification at the 1-year post-treatment follow-up.

Ethical approval for this study (application 03.36) was granted by the National Health Service Local Research Ethics Committee (NHS-LREC) of St Mary's Hospital (London, UK) and the Uganda National Council of Science and Technology (Kampala, Uganda). Written informed consent was obtained from the head teachers of each school, and oral informed consent was obtained from each child before fingerprick blood and stool samples were collected and anthelminthic treatments were administered.

\subsection{Data management and statistical analyses}

Analyses were performed with Minitab version 14 (Minitab Inc., State College, PA, USA) and STATA version 8.0 (Stata Corp., College Station, TX, USA). The frequency distributions of the district-level ACRs were assessed visually with SAS version 8 (SAS Institute Inc., Campus Drive, NC, USA). Sensitivity and specificity were calculated to assess the general diagnostic performance of the ACR in predicting S. mansoni infection. To estimate an appropriate binary cut-off of ACR scores between infection-positive or -negative children, a receiver operating characteristic (ROC) curve was plotted.

To investigate further possible explanatory variables for ACR scores, all variables were explored individually by univariate linear regression. Those variables with a $P$-value $<0.2$ were included in a multivariate analysis. Covariates or factors with a $P$-value $>0.05$ were removed from this model in a stepwise backward elimination approach. The haemoglobin level and the natural logs of (SMEPG + 1) and $(H W E P G+1)$ and an interaction term between these two covariates were included in the original model. Age, sex and the interaction between them, as well as children's height and the school attended, were also included. 


\section{Results}

\subsection{Study compliance and operational results}

There were 4354 school children enrolled in the study at baseline, of whom 4189 (96.2\%) had complete data sets with ACRs and status of parasitic infection (i.e. S. mansoni, A. lumbricoides, T. trichiura and hookworms). Oneyear post treatment, 2748 (63.1\%) of those who were present at the baseline survey were re-examined. After matching the unique identification numbers, a total of 2644 children had complete data sets at both baseline and the 1-year posttreatment follow-up surveys, giving an overall compliance of $60.7 \%$. There were 1351 boys (51.1\%) and 1293 girls (48.9\%). At baseline, 560 of these children ( 284 boys and 276 girls) also had ultrasound examinations.

\subsection{Parasitic infections}

Table 1 summarises the parasitological findings from the baseline and 1-year post-treatment surveys. Microscopic examination of a single Kato-Katz thick film during baseline revealed a S. mansoni infection prevalence of $44.3 \%$. Prevalences of hookworms, $A$. lumbricoides and $T$. trichiura were $51.8 \%, 2.4 \%$ and $2.4 \%$, respectively. Other parasitic infections were observed in $1.6 \%$ of the children surveyed, including Enterobius vermicularis and Hymenolepis nana. Polyparasitism was relatively common, with 703 (26.6\%) of the children harbouring two or more parasites concurrently. In view of the low prevalence of $A$. lumbricoides, $E$. vermicularis, $H$. nana and $T$. trichiura, these data were excluded from further analyses.

At baseline, S. mansoni prevalence did not show a significant association with sex (boys $44.7 \%$ vs. girls $43.9 \%$; $\chi^{2}=0.16$, d.f. $=1, P=0.687$ ) but it did with age; older children were significantly more likely to be infected with S. mansoni than their younger counterparts (age 6 years, $36.3 \%$; age 7 years, $42.9 \%$; age 8 years, $44.4 \%$; age 11 years, 52.6\%; $\chi^{2}=35.50$, d.f. $=3, P<0.001$ ). Similarly, the prevalence of hookworms was not significantly associated with sex (boys $53.5 \%$ vs. girls $50.0 \% ; \chi^{2}=3.20$, d.f. $=1, P=0.074$ ) but was with age (age 6 years, $48.7 \%$; age 7 years, 48.6\%; age 8 years, $52.0 \%$; age 11 years,
57.0\%; $\chi^{2}=13.68$, d.f. $\left.=3, P=0.003\right)$. Anaemia, defined as haemoglobin $<11 \mathrm{~g} / \mathrm{dl}$ (Crawley, 2004), was recorded in 909 children $(34.4 \%)$, the prevalence of which was not significantly associated with sex (boys $35.2 \%$ vs. girls $34.3 \%$; $\chi^{2}=0.27$, d.f. $=1, P=0.605$ ), but it decreased significantly with age (age 6 years, $42.2 \%$; age 7 years, $38.3 \%$; age 8 years, $34.2 \%$; age 11 years, $25.4 \%$; $\chi^{2}=44.58$, d.f. $=3, P<0.001$ )

At the 1-year post-treatment survey, examination of a single Kato-Katz thick film showed that the prevalence of S. mansoni had decreased to $29.2 \%$ (34\% reduction), which was highly significant $\left(\chi^{2}=563.91\right.$, d.f. $\left.=1, P<0.001\right)$. The geometric mean infection intensity of $S$. mansoni decreased from 216.4 EPG to 109.8 EPG (49\% reduction). The prevalence of hookworm infection decreased significantly to $24.0 \%$ (54\% reduction; $\chi^{2}=90.73$, d.f. $=1, P<0.001$ ), whilst that of anaemia decreased significantly to $30.7 \% \quad(10.8 \%$ reduction; $\chi^{2}=193.05$, d.f. $=1, P<0.001$ ).

\subsection{Distribution of ACRs}

The three children presenting with particularly grossly distended abdomens depicted in Figure 2 are illustrative of referrals to hospitals. The ACR of each child was $\geq 1.05$. More generally, ACR scores at baseline appeared to follow a normal distribution (Figure $3 \mathrm{~A}$ ).

The ACR scores are shown in Table 2. The arithmetic mean ACR was significantly lower in boys $(0.936)$ than in girls $(0.947 ;$ t -test statistic $=5.71$, d.f. $=2643, P<0.001)$ and decreased significantly with age (age 6 years, 0.957 ; age 7 years, 0.945; age 8 years, 0.940; age 11 years, 0.927; $t$-test statistic $=-10.32$, d.f. $=2643, P<0.001)$. Boys had a greater range of values $(0.72-1.28)$ than girls $(0.76-1.18)$ and a greater SD from the mean (boys 0.056 vs. girls 0.047 ). A total of $10.7 \%$ and $2.5 \%$ of the school children were observed to have $A C R s \geq 1.0$ and $\geq 1.05$, respectively.

Analysis of the ACR scores in each of the eight districts showed a significant difference between districts $(P<0.05$; Figure $3 \mathrm{~B}$ ). In particular, the frequency distribution of the ACRs in Masindi was significantly different to that of the other seven districts, whilst there were no significant differences in the distribution of ACRs within Arua, Busia, Moyo and Nebbi districts or within Bugiri, Hoima and Mayuge districts. The prevalence of ACR scores $\geq 1.0$ appeared to be overdispersed between districts (Figure 4A), with notable

Table 1 Parasitological findings at baseline and at 1-year post-treatment follow-up

\begin{tabular}{|c|c|c|c|c|c|c|c|c|c|c|}
\hline \multirow[t]{2}{*}{ Variable } & \multicolumn{5}{|l|}{ Baseline } & \multicolumn{5}{|c|}{ 1-year follow-up } \\
\hline & $\begin{array}{l}\text { Overall } \\
(\%)\end{array}$ & $\begin{array}{l}\text { Male }(\%) \\
(n=1351)\end{array}$ & $\begin{array}{l}\text { Female }(\%) \\
(n=1293)\end{array}$ & $x^{2}$ & $P$-value & $\begin{array}{l}\text { Overall } \\
(\%)\end{array}$ & $\begin{array}{l}\text { Male }(\%) \\
(n=1351)\end{array}$ & $\begin{array}{l}\text { Female }(\%) \\
(n=1293)\end{array}$ & $x^{2}$ & $P$-value \\
\hline Schistosoma mansoni & 44.3 & 44.7 & 43.9 & 0.16 & 0.687 & 29.2 & 31.1 & 27.2 & 4.77 & 0.029 \\
\hline Hookworms & 51.8 & 53.5 & 50.0 & 3.20 & 0.074 & 24.0 & 26.9 & 21.0 & 12.66 & $<0.001$ \\
\hline Ascaris lumbricoides & 2.4 & 2.7 & 2.1 & 1.18 & 0.277 & 1.7 & 1.6 & 1.8 & 0.20 & 0.652 \\
\hline Trichuris trichiura & 2.4 & 2.4 & 2.3 & 0.04 & 0.837 & 2.4 & 2.3 & 2.5 & 0.09 & 0.761 \\
\hline Other parasites & 1.6 & 1.9 & 1.2 & 2.97 & 0.085 & 0.4 & 0.3 & 0.8 & 1.79 & 0.181 \\
\hline Polyparasitism & 26.6 & 28.0 & 25.2 & 2.74 & 0.098 & 10.4 & 11.8 & 9.1 & 5.23 & 0.022 \\
\hline None of above & 26.6 & 25.5 & 27.7 & 1.68 & 0.195 & 58.8 & 57.1 & 60.6 & 3.47 & 0.063 \\
\hline Anaemia $^{a}$ & 34.4 & 35.2 & 34.3 & 0.27 & 0.605 & 30.7 & 32.2 & 29.1 & 3.02 & 0.082 \\
\hline
\end{tabular}

\footnotetext{
a Haemoglobin $<11 \mathrm{~g} / \mathrm{dl}$.
} 


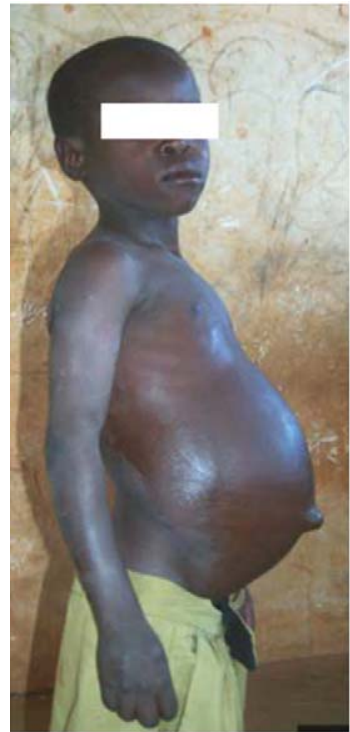

(A)

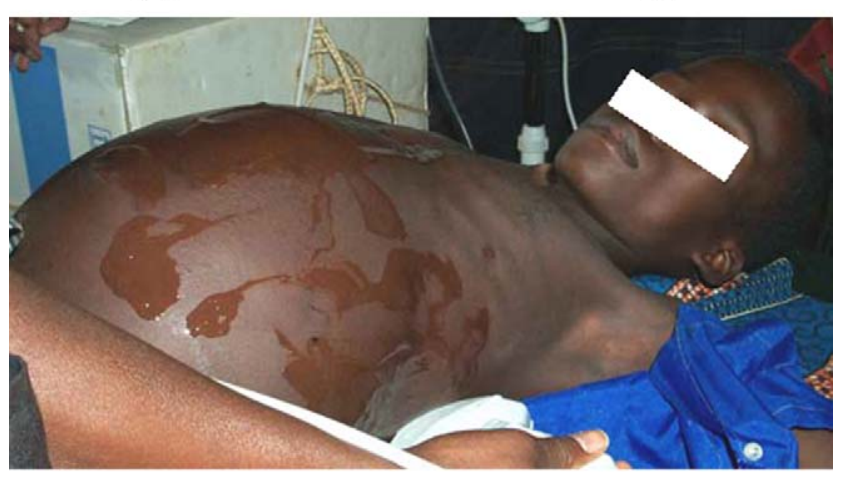

(C)

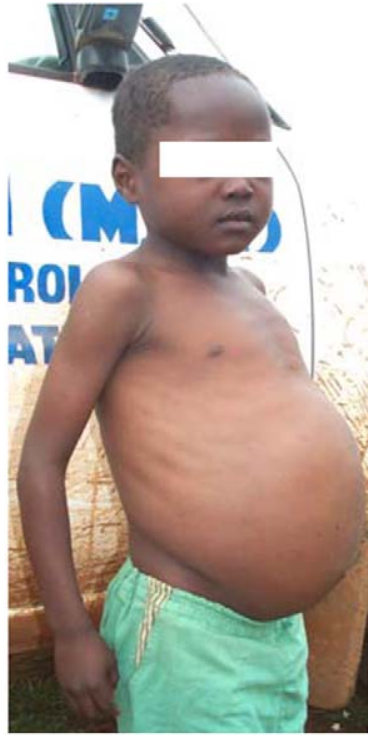

(B)

Figure 2 Three children with grossly distended abdomens, i.e. abdominal circumference ratio $(A C R) \geq 1.05$, encountered during the surveys. (A) An 8-year-old child with an ACR score of 1.06 , having obvious ventral collaterals and advanced liver fibrosis as seen on ultrasound (Niamey pattern D). Whilst this child was Schistosoma mansoni egg-negative upon initial Kato-Katz examination, formol-ether concentration of the stool sample revealed the presence of calcified S. mansoni eggs. (B) An 11-year-old boy with an ACR of 1.12; ultrasound examination revealed a diffuse echogenic liver. (C) A 14-year-old child encountered at Bukizibu primary school in Hoima district, with very severe abdominal distension $(A C R \sim 1.3)$ who was immediately referred to Hoima hospital and who subsequently died. increases in Bugiri, Hoima and Mayuge, with six schools in these districts having a prevalence of ACR scores $\geq 1.0$ of $20 \%$ and above, namely Kibanjwa and Runga in Hoima district, Bukizibu and Bugoto Lake View in Mayuge district, and Buyondo and Kibuye in Bugiri district (Figure 4B).

An individual-level preliminary univariate analysis showed that the arithmetic mean ACR was slightly higher among those school children with S. mansoni infection than in those without $(0.944$ vs. 0.940$)$, which was statistically significant $(t$-test statistic $=2.01$, d.f. $=2643, P=0.044)$. At the school level, although the mean ACR levels increased with $S$. mansoni prevalence and geometric mean infection intensity (Figures 5A and B), the prevalence of ACR scores $\geq 1.0$ failed to show a clear relationship (Figure $5 C$ ). There was no significant difference in the arithmetic mean ACR of hookworm-positive and -negative children, whilst the ACR of anaemic children was significantly higher than nonanaemic children $(0.945$ vs. $0.940 ; t$-test statistic $=2.63$, d.f. $=2643, P=0.008$ ). From the ROC analysis, an ACR score of 0.925 obtained the highest sensitivity $(65.7 \%)$ and specificity (40.1\%) for diagnosis of S. mansoni infection, and similar values were obtained upon consideration of moderate to heavy infection intensities. At the individual level, the performance of the ACR for general diagnosis of S. mansoni infection was shown to be poor.

\subsection{General linear modelling}

The model with the best fit was chosen on the basis of the highest observed $r^{2}$ score. This model showed that haemoglobin level, age, sex, height, natural log of $(S M E P G+1)$ and school location may be used to predict or explain variation in the ACR at a $P$-value of 0.05 . Hookworm data (natural log of (HWEPG +1$)$ or a binary classification of infection status) were not found to be significant. In justification of this final model, an analysis of the residuals confirmed assumptions of normality.

\subsection{One-year post-treatment follow-up}

No evidence of bias was found in terms of $\operatorname{sex}\left(\chi^{2}=0.02\right.$, d.f. $=1, P=0.889)$ or age $\left(\chi^{2}=3.92\right.$, d.f. $\left.=3, P=0.270\right)$ for the children who dropped out (Table 3 ). Moreover, odds ratios of the variables inspected revealed no obvious dropout bias (Table 4). Both overall prevalence and intensity of S. mansoni infections were lower 1-year post-treatment compared with the baseline survey (Table 1). In parallel, there was measurable change in the ACR arithmetic means by district and school (Table 2; Figure 6). The extreme ACR

Table 2 Mean abdominal circumference ratio (ACR) scores and percent prevalence of $A C R \geq 1.0$ at baseline and at 1 -year post-treatment follow-up

\begin{tabular}{lllll}
\hline & \multicolumn{2}{l}{ ACR scores } & & \\
\cline { 2 - 4 } & Male $(n=1351)$ & Female $(n=1293)$ & Total & \\
\hline Baseline & 0.936 & 0.947 & 0.942 & $10.7(n=284)$ \\
1-year follow-up & 0.946 & 0.953 & 0.949 & $11.4(n=302)$ \\
\hline
\end{tabular}




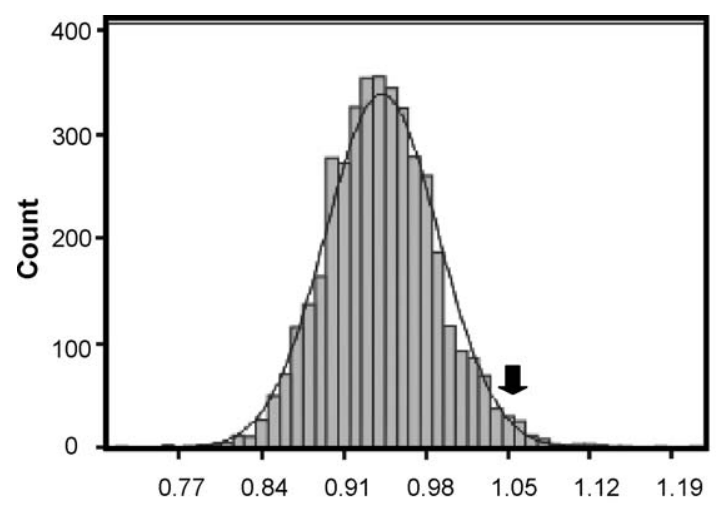

(A)

ACR

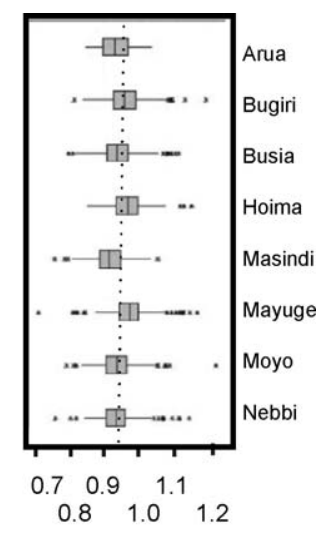

(B) ACR

Figure 3 Overall frequency distribution of abdominal circumference ratio (ACR) scores and box plot of ACR scores grouped by district. (A) The distribution of ACR scores appears to be normal; values $>1.05$ might identify children with similar signs to the child shown in Figure 2A. (B) Box plot of ACR scores by district, which reveals geographic variation. Of particular note is the occurrence of children with elevated ACR scores as statistical outliers, potentially having very serious abdominal distension.

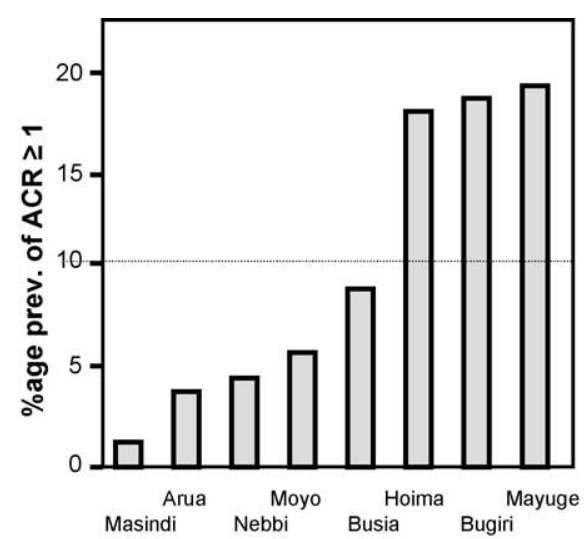

(A)

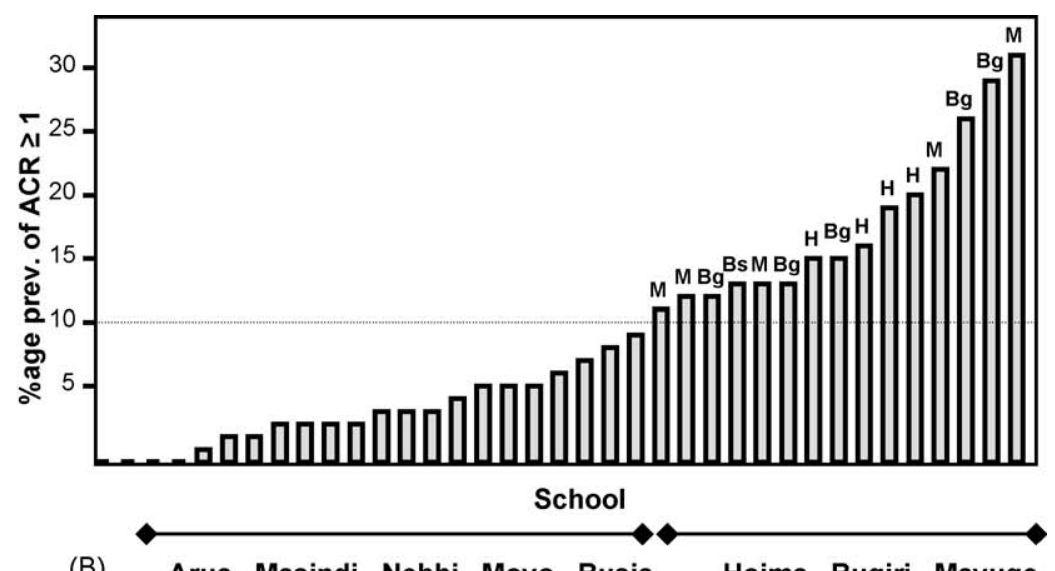

(B)

Arua, Masindi, Nebbi, Moyo, Busia

Hoima, Bugiri, Mayuge

Figure 4 The prevalence of abdominal circumference ratio (ACR) $\geq 1.0$ exhibits geographical overdispersion. (A) Prevalence of $A C R \geq 1.0$ scores were much elevated in the three districts of Bugiri, Hoima and Mayuge (dotted line denotes mean prevalence). (B) Prevalence of $A C R \geq 1.0$ by school; note that several schools have an elevated prevalence and, of the 15 schools with higher than average prevalences, one school, Bwaniha, originates from Busia (Bs) whilst the remainder are within Hoima $(H)$, Mayuge $(M)$ and Bugiri (Bg) districts.

values seen at baseline were not found at the 1 -year followup (baseline $0.72-1.28$ vs. 1-year follow-up 0.77-1.17). High initial values tended to decrease whereas low initial values tended to increase over time, exhibiting regression towards the mean ( $t$-test statistic $=-9.71$, d.f. $=2643$,

Table 3 Age and sex profile of children lost from the study cohort

\begin{tabular}{llll}
\hline $\begin{array}{l}\text { Initial cohort } \\
\text { age (years) }\end{array}$ & \multicolumn{3}{l}{ Composition of drop-outs } \\
\cline { 2 - 4 } & $\begin{array}{l}\text { Male (\%) } \\
(n=786)\end{array}$ & $\begin{array}{l}\text { Female (\%) } \\
(n=759)\end{array}$ & $\begin{array}{l}\text { Overall } \\
(\%)\end{array}$ \\
\hline 6 & 47.1 & 53.4 & 26.4 \\
7 & 51.1 & 48.9 & 22.8 \\
8 & 51.5 & 48.5 & 24.0 \\
11 & 53.9 & 46.1 & 26.8 \\
\hline
\end{tabular}

$P<0.001)$. Despite these changes, the overall prevalence of $A C R \geq 1.0$ remained at $11.4 \%$ and was still overdispersed between districts. After standardising the change in ACR with height, a mixed modelling approach revealed that fixed

Table 4 Selected variables with associated odds ratios for children lost from the cohort

\begin{tabular}{ll}
\hline Variable & Odds ratio $(95 \% \mathrm{Cl})$ \\
\hline $\mathrm{ACR} \geq 1.00$ & $1.11(0.91-1.36)$ \\
$\mathrm{ACR} \geq 1.05$ & $1.17(0.73-1.85)$ \\
Infected with Schistosoma mansoni & $1.09(0.96-1.24)$ \\
$\quad(E P G>0)$ & \\
Infected with S. mansoni $(\mathrm{EPG} \geq 100)$ & $1.12(0.98-1.29)$ \\
Infected with hookworm & $0.99(0.92-1.07)$ \\
Anaemia (haemoglobin $<11 \mathrm{~g} / \mathrm{dl})$ & $0.97(0.85-1.11)$ \\
\hline
\end{tabular}

ACR: abdominal circumference ratio; EPG, eggs per gram of faeces. 

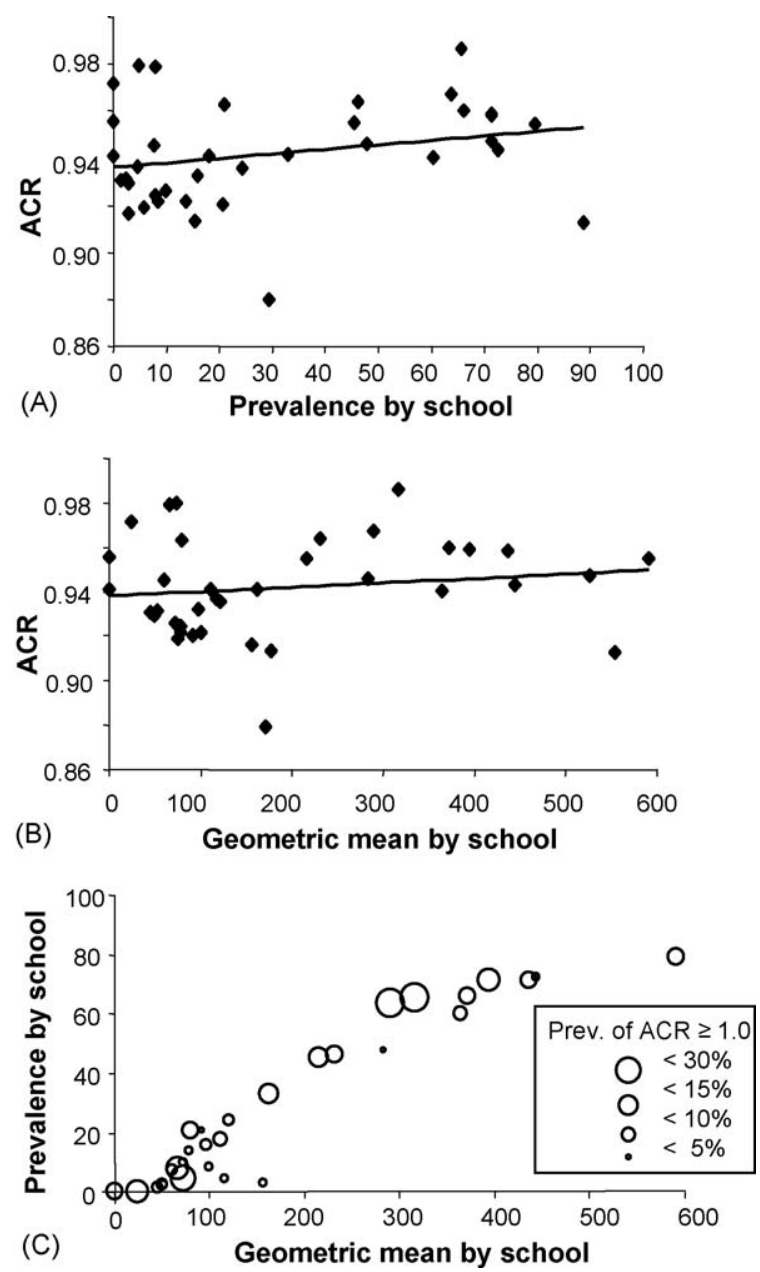

Figure 5 (A) Scatter plot of arithmetic abdominal circumference ratio (ACR) mean by school against Schistosoma mansoni prevalence by school (trend line $y=2.0 \mathrm{E}^{-4} x+0.9361$ ). (B) Scatter plot of arithmetic ACR mean by school against $S$. mansoni geometric mean infection intensity by school (trend line $y=2.0 E^{-5} x+0.9381$ ). (C) Bubble chart of relationship between prevalence of $A C R \geq 1.0$ by school with $S$. mansoni prevalence and geometric mean infection intensity by school. Relationships between ACR and S. mansoni infection prevalence and intensity show a weak positive association. In contrast, no clear relationship could be seen between the prevalence of $A C R \geq 1.0$ by school with S. mansoni prevalence and intensity.

effects of haemoglobin scores and (HWEPG + 1) had a significant effect on ACR at the $5 \%$ level, whilst $(S M E P G+1)$ did not.

\section{Discussion}

At the individual level, the distended abdomen, indicative of hepatosplenomegaly and portal hypertension, can be a striking visual sign of advanced morbidity due to S. mansoni, S. japonicum or S. mekongi infection (Biays et al., 1999; Peters and Gilles, 1995). From an epidemiological perspective, however, the distribution and extent of this condition within the school-age community, where it likely develops, has only been scantly documented (Ongom et al., 1972).
Like the children depicted in Figure 2, those with elevated ACR scores (i.e. $\geq 1.05$ ) could be considered to be sufferers of distended abdomen syndrome, identifying $2.5 \%(n=67)$ of the children in our baseline sample as potentially quite moribund.

Over 30 years ago at Panyagoro (see Figure 1), Ongom et al. (1972) found nearly $14 \%$ of the children within their community cohort to have abdominal distension and ascites, judged by visual presentation and auscultation methods, respectively. The ACR metric may provide a more direct quantification of pathognomonic signs (as evidenced by the children shown in Figure 2) and enables convenient comparison between children. In accordance with recent findings (Vennervald et al., 2005), a high proportion (67.8\%) of the children in our sample had firm and $>1 \mathrm{~cm}$ enlarged livers and spleens, with several showing surprisingly advanced liver fibrosis on ultrasound. Although the distribution of the ACRs across the sample appeared normal (see Figure 3), further analysis of ACR scores revealed geographic overdispersion within Bugiri, Hoima and Mayuge districts and pinpointed several schools where morbidity appeared much elevated (Figure 4).

This geographic overdispersion was attributable to elevated ACR scores within nine schools where the prevalence of $A C R \geq 1.0$ was in excess of $15 \%$, and previous studies have identified these three districts as having generally higher prevalence and intensity of $S$. mansoni infection (Kabatereine et al., 2004a). As infections are generally lighter in Arua and Moyo, and there has been previous mass distribution of anthelminthics in Masindi and Nebbi, this might shed some light on the general patterns of lower ACR scores. Overdispersion of morbidity associated with intestinal schistosomiasis in Uganda is perhaps not unexpected given that geographic heterogeneity is known elsewhere (Boisier et al., 2001; van der Werf et al., 2002). Moreover, as this survey was based within schools, children too sick to attend class will have been missed, so the true prevalence of this syndrome within the school-age community is likely to be even higher. The serious consequences of schistosomiasis reported by Ongom et al. (1972) are still in evidence today, as demonstrated by the subsequent death of the child depicted in Figure $2 \mathrm{C}$.

At the group or school level, uncovering the precise aetiology that gave rise to elevated ACR scores was problematic. Although the mean ACR score was slightly higher among school children with $S$. mansoni infection than those without it, Figure 5 illustrates that a more general relationship between elevated ACR scores with prevalence (Figure 5A) and intensity (Figure $5 B$ ) of S. mansoni infection was not immediately apparent. In addition, Figure $5 \mathrm{C}$ shows that a prevalence of $A C R \geq 1.0$ may also occur in certain schools where general prevalence and intensity of S. mansoni was low.

Concurrent infections with several other parasites or pathogens, the most important of which may be malaria, could also be key factors affecting ACR scores, although Ongom et al. (1972) ruled out hepatitis of viral origin. As both malaria and schistosomiasis can be acquired in early childhood and produce overlapping physical signs (Booth et al., 2004a; Mwatha et al., 2003; Warrell, 2002), and as areas characterised by high $S$. mansoni prevalence may also coincide with malarious regions (Vennervald and Dunne, 2004), 

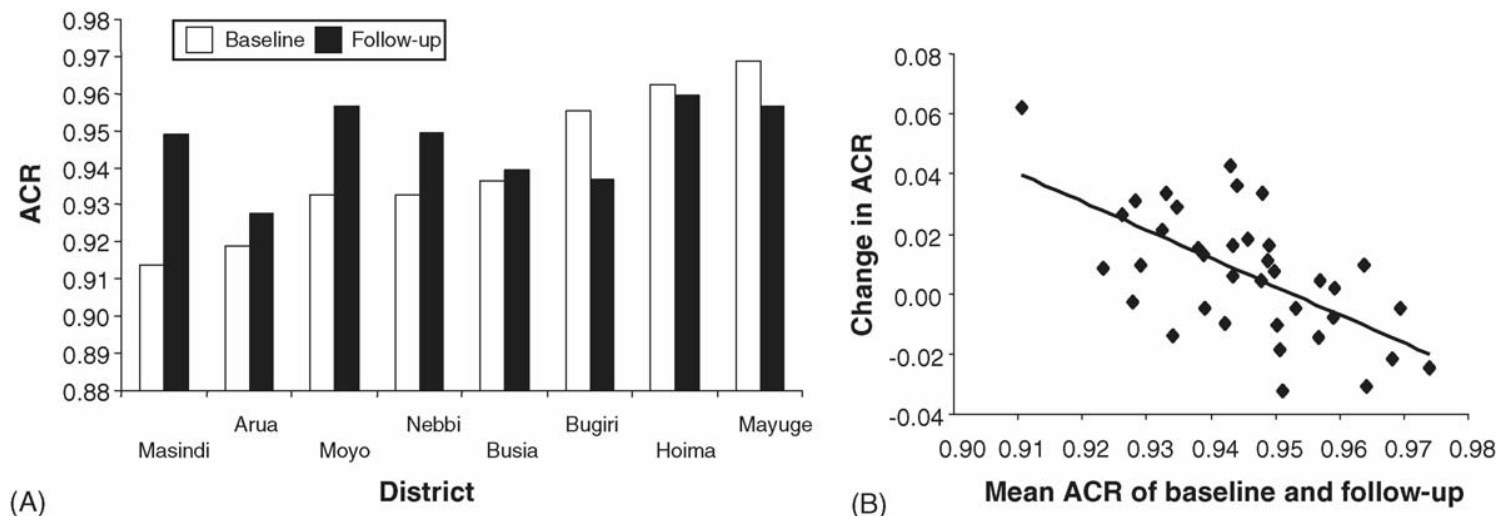

Figure 6 Changes in abdominal circumference ratio (ACR) scores between baseline and 1-year post-treatment follow-up show putative ameliorative effects of anthelminthic treatment by general regression towards the mean. (A) Bar chart of ACR scores by district at baseline and at 1-year follow-up. Decreases were seen in districts with elevated ACRs at baseline whilst increases were seen in districts with depressed ACRs. (B) Scatter plot of the change in ACRs by school at baseline and at 1-year follow-up. Note the general left-to-right trend indicating regression of ACR scores towards the mean (trend line $y=-0.942 x+0.8975$ ).

it is difficult to assess directly the relative roles of either disease in influencing ACR scores. Similarly, nutritional factors might also determine ACR scores. Whilst lower haemoglobin levels were a significant factor, these may have been confounded by parasitism (Olsen et al., 1998), but certainly malnutrition, particularly that giving rise to kwashiorkorlike signs, could contribute to an increased ACR. A possible way forward may be to take other biometrical measures such as mid-upper arm circumference and attempt to correlate this variable with ACR scores, which perhaps might better identify chronically malnourished children not apparent from a weight-for-height Z-score. In addition, host genetic or immunological factors, the presence of environmental toxins, or genetic differences in S. mansoni may also be involved (Abel and Dessein, 1997; Booth et al., 2004b), all of which may individually or collectively play a role. It will remain difficult to disentangle the relative interplay of these co-factors, but it is evident that the relationship between single point prevalence/intensity measures and chronically evolved morbidity as detected during epidemiological surveys is not always obvious (Booth et al., 2004a).

Recovery of the cohort 1-year post treatment did not appear to have a sex or age bias or a strong association with helminth infection or anaemia (see Tables 2 and 3). Although the odds ratios for children with ACR scores $\geq 1.0$ might be considered to be slightly higher than for the other children, given the associated $95 \% \mathrm{Cl}$ there was no indication of statistical significance. In total, nearly $40 \%$ of the children were no longer available for the 1-year follow-up, demonstrating the general instability of regular primary school attendance in these areas. The putative ameliorative effects of anthelminthic treatment, however, could be seen upon comparison of ACR scores between baseline and 1-year posttreatment follow-up data. A negative correlation between the change in $A C R\left(A C R_{1}-A C R_{0}\right)$ and the mean of the two ACR values $\left(0.5\left[A C R_{0}+A C R_{1}\right]\right)$ showed a tendency of higher mean ACR scores by school to show a larger decrease than those with lower mean ACR scores. The ameliorative effect of treatment on reduction of organomegaly is well known
(Boisier et al., 1998; Vennervald et al., 2005). However, regression towards the mean can also be a general statistical phenomenon of a reduced sample size. As the children in the Ugandan cohort will be followed for a further 2 years, it is conceivable that a clearer picture might emerge but the diminishing cohort recovery is of growing concern. Repeated cross-sectional surveys, rather than a cohort design, might therefore be better given the known yearly rate of cohort attrition.

To conclude, infection with S. mansoni is one of the likely explanatory variables for distended abdomen syndrome in Ugandan children, but the serious nature of the condition and geographic aggregations in Bugiri, Hoima and Mayuge districts are of concern. Increased surveillance and monitoring of children with elevated ACR scores is therefore strongly advised and in future this should also attempt to include more detailed quantification of the attributable fractions of parasitic infections, especially that of Plasmodium spp., and malnutrition. A more general inspection of humoral biomarkers that give rise to or are associated with this condition should be worthwhile (Booth et al., 2004b; Wang et al., 2004).

\section{Conflicts of interest statement}

The authors have no conflicts of interest concerning the work reported in this paper.

\section{Acknowledgements}

We are grateful to the school teachers, parents and children of Uganda who participated in this study. We would also like to thank the dedicated technicians of Vector Control Division, Kampala, who provided diligent technical assistance while in the field, especially to Richard Galimaka who took the ACR measurements. Funding from the Bill and Melinda Gates Foundation was gratefully received. J. Utzinger acknowledges financial support from the Swiss National Science Foundation (Project No. PPOOB-102883). The manuscript much benefited from the helpful suggestions of the anonymous referees. 


\section{References}

Abel, L., Dessein, A.J., 1997. The impact of host genetics on susceptibility to human infectious diseases. Curr. Opin. Immunol. 9, 509-516.

Biays, S., Stich, A.H.R., Odermatt, P., Long, C., Yersin, C., Men, C., Saem, C., Lormand, J.D., 1999. [A foci of Schistosoma mekongi rediscovered in northeast Cambodia: cultural perception of the illness; description and clinical observation of 20 severe cases.] Trop. Med. Int. Health 4, 662-673 [in French].

Boisier, P., Ramarokoto, C.E., Ravaoalimalala, V.E., Rabarijaona, L., Serieye, J., Roux, J., Esterre, P., 1998. Reversibility of Schistosoma mansoni-associated morbidity after yearly mass praziquantel therapy: ultrasonographic assessment. Trans. R. Soc. Trop. Med. Hyg. 92, 451-453.

Boisier, P., Ramarokoto, C.E., Ravoniarimbinina, P., Rabarijaona, L., Ravaoalimalala, V.E., 2001. Geographic differences in hepatosplenic complications of schistosomiasis mansoni and explanatory factors of morbidity. Trop. Med. Int. Health 6, 699-706.

Booth, M., Vennervald, B.J., Kabatereine, N.B., Kazibwe, F., Ouma, J.H., Kariuki, C.H., Muchiri, E., Kadzo, H., Ireri, E., Kimani, G., Mwatha, J.K., Dunne, D.W., 2004a. Hepatosplenic morbidity in two neighbouring communities in Uganda with high levels of Schistosoma mansoni infection but very different durations of residence. Trans. R. Soc. Trop. Med. Hyg. 98, 125-136.

Booth, M., Mwatha, J.K., Joseph, S., Jones, F.M., Kadzo, H., Ireri, E., Kazibwe, F., Kemijumbi, J., Kariuki, C., Kimani, G., Ouma, J.H., Kabatereine, N.B., Vennervald, B.J., Dunne, D.W., 2004b. Periportal fibrosis in human Schistosoma mansoni infection is associated with low IL-10, low IFN- $\gamma$, high TNF- $\alpha$, or low RANTES, depending on age and gender. J. Immunol. 172, 1295-1303.

Brooker, S., Whawell, S., Kabatereine, N.B., Fenwick, A., Anderson, R.M., 2004. Evaluating the epidemiological impact of national control programmes for helminths. Trends Parasitol. 20, 537-545.

Crawley, J., 2004. Reducing the burden of anemia in infants and young children in malaria-endemic countries of Africa: from evidence to action. Am. J. Trop. Med. Hyg. 71 (Suppl. 2), 25-34.

Engels, D., Chitsulo, L., Montresor, A., Savioli, L., 2002. The global epidemiological situation of schistosomiasis and new approaches to control and research. Acta Trop. 82, 139-146.

Fenwick, A., Savioli, L., Engels, D., Bergquist, N.R., Todd, M.H., 2003. Drugs for the control of parasitic diseases: current status and development in schistosomiasis. Trends Parasitol. 19, 509-515.

Hatz, C.F.R., 2001. The use of ultrasound in schistosomiasis. Adv. Parasitol. 48, 225-284.

Kabatereine, N.B., Odongo-Aginya, E.I., Lakwo, L.T., 1996. Schistosoma mansoni along Lake Albert, Kibale District, western Uganda. East Afr. Med. J. 73, 502-504.

Kabatereine, N.B., Brooker, S., Tukahebwa, E.M., Kazibwe, F., Onapa, A.W., 2004a. Epidemiology and geography of Schistosoma mansoni in Uganda: implications for planning control. Trop. Med. Int. Health 9, 372-380.

Kabatereine, N.B., Kemijumbi, J., Ouma, J.H., Kariuki, H.C., Richter, J., Kadzo, H., Madsen, H., Butterworth, A.E., Ornbjerg, N., Vennervald, B.J., 2004b. Epidemiology and morbidity of Schistosoma mansoni infection in a fishing community along Lake Albert in Uganda. Trans. R. Soc. Trop. Med. Hyg. 98, 711-718.

Kabatereine, N.B., Tukahebwa, E., Kazibwe, F., Namwangye, H., Zaramba, S., Brooker, S., Stothard, J.R., Kamenka, C., Whawell, S., Webster, J.P., Fenwick, A., 2006. Progress towards countrywide control of schistosomiasis and soil-transmitted helminthiasis in Uganda. Trans. R. Soc. Trop. Med. Hyg. 100, 208-215.

Katz, N., Chavez, A., Pellegrino, J., 1972. A simple device for quantitative stool thick smear technique in schistosomiasis mansoni. Rev. Inst. Med. Trop. Sao Paulo 14, 397-400.
King, C.H., Magak, P., Salam, E.A., Ouma, J.H., Kariuki, H.C., Blanton, R.E., 2003. Measuring morbidity in schistosomiasis mansoni: relationship between image pattern, portal vein diameter and portal branch thickness in large-scale surveys using new WHO coding guidelines for ultrasound in schistosomiasis. Trop. Med. Int. Health 8, 109-117.

King, C.H., Dickman, K., Tisch, D.J., 2005. Reassessment of the cost of chronic helmintic infection: a meta-analysis of disability related outcomes in endemic schistosomiasis. Lancet 365 , 1561-1569.

Kongs, A., Marks, G., Verle, P., Van der Stuyft, P., 2001. The unreliability of the Kato-Katz technique limits its usefulness for evaluating Schistosoma mansoni infections. Trop. Med. Int. Health 6, 163-169.

Lambertucci, J.R., Serufo, J.C., Gerspacher-Lara, R., Rayes, A.A., Teixeira, R., Nobre, V., Antunes, C.M., 2000. Schistosoma mansoni: assessment of morbidity before and after control. Acta Trop. 77, 101-109.

Montresor, A., Odermatt, P., Muth, S., Iwata, F., Raja'a, Y.A., Assis, A.M., Zulkifli, A., Kabatereine, N.B., Fenwick, A., Al-Awaidy, S., Allen, H., Engels, D., Savioli, L., 2005. The WHO dose pole for the administration of praziquantel is also accurate in non-Africa populations. Trans. R. Soc. Trop. Med. Hyg. 99, 78-81.

Mwatha, J.K., Jones, F.M., Mohamed, G., Naus, C.W., Riley, E.M., Butterworth, A.E., Kimani, G., Kariuki, C.H., Ouma, J.H., Koech D., Dunne, D.W., 2003. Associations between anti-Schistosoma mansoni and anti-Plasmodium falciparum antibody responses and hepatosplenomegaly, in Kenyan schoolchildren. J. Infect. Dis. 187, 1337-1341.

Nooman, Z.M., Hassan, A.H., Mishrirky, A.M., Ragheb, M., AbuSaif, A.N., Abaza, S.M., Serwah, A.A., Kamal, M., Fouad, M. 1995. The use and limitations of ultrasonography in the diagnosis of liver morbidity attributable to Schistosoma mansoni infection in community-based surveys. Mem. Inst. Oswaldo Cruz 90, 147-154.

Olsen, A., Magnussen, P., Ouma, J.H., Andreassen, J., Friis, H., 1998. The contribution of hookworm and other parasitic infections to haemoglobin and iron status among children and adults in western Kenya. Trans. R. Soc. Trop. Med. Hyg. 92, 643649.

Ongom, V.L., Bradley, D.J., 1972. The epidemiology and consequences of Schistosoma mansoni infection in West Nile, Uganda. I. Field studies of a community at Panyagoro. Trans. R. Soc. Trop. Med. Hyg. 66, 835-851.

Ongom, V.L., Owor, R., Grundy, R., Bradley, D.J., 1972. The epidemiology and consequences of Schistosoma mansoni infection in West Nile, Uganda. II. Hospital investigation of a sample from Panyagoro community. Trans. R. Soc. Trop. Med. Hyg. 66, 852-863.

Peters, W., Gilles, H.M., 1995. Color Atlas of Tropical Medicine and Parasitology, fourth ed. Mosby-Wolfe, London.

Raso, G., Utzinger, J., Silué, K.D., Ouattara, M., Yapi, A., Toty, A., Matthys, B., Vounatsou, P., Tanner, M., N'Goran, E.K., 2005. Disparities in parasitic infections, perceived ill health and access to health care among poorer and less poor schoolchildren of rural Côte d'Ivoire. Trop. Med. Int. Health 10, 42-57.

Ruiz, R., Candia, P., Garassini, M., Tombazzi, C., Certad, G., Bruces, A.C., Noya, O., Alarcon de Noya, B., 2002. Schistosomiasis mansoni in low transmission areas: abdominal ultrasound. Mem. Inst. Oswaldo Cruz 97 (Suppl. 1), 153-159.

Utzinger, J., Keiser, J., 2004. Schistosomiasis and soil-transmitted helminthiasis: common drugs for treatment and control. Expert Opin. Pharmacother. 5, 263-285.

van der Werf, M.J., de Vlas, S.J., Looman, C.W.N., Nagelkerke, N.J.D., Habbema, J.D.F., Engels, D., 2002. Associating community prevalence of Schistosoma mansoni infection with prevalence of signs and symptoms. Acta Trop. 82, 127137. 
van der Werf, M.J., de Vlas, S.J., Brooker, S., Looman, C.W.N. Nagelkerke, N.J.D., Habbema, J.D.F., Engels, D., 2003. Quantification of clinical morbidity associated with schistosome infection in sub-Saharan Africa. Acta Trop. 86, 125-139.

Vennervald, B.J., Dunne, D.W., 2004. Morbidity in schistosomiasis: an update. Curr. Opin. Infect. Dis. 17, 439-447.

Vennervald, B.J., Booth, M., Butterworth, A.E., Kariuki, H.C., Kadzo, H., Ireri, E., Amaganga, C., Kimani, G., Kenty, L., Mwatha, J., Ouma, J.H., Dunne, D.W., 2005. Regression of hepatosplenomegaly in Kenyan school-aged children after praziquantel treatment and three years of greatly reduced exposure to Schistosoma mansoni. Trans. R. Soc. Trop. Med. Hyg. 99, 150-160.

Wang, Y., Holmes, E., Nicholson, J.K., Cloarec, O., Chollet, J., Tanner, M., Singer, B.H., Utzinger, J., 2004. Metabonomic investi- gations in mice infected with Schistosoma mansoni: an approach for biomarker identification. Proc. Natl. Acad. Sci. USA 101, 12676-12681.

Warrell, D.A., 2002. Clinical features of malaria, in: Warrell, D.A., Gilles, H.M. (Eds), Essential Malariology, fourth ed. Arnold, London, pp. 191-204.

WHO, 2002. Prevention and control of schistosomiasis and soiltransmitted helminthiasis: report of a WHO expert committee. World Health Organization, Geneva, Technical Report Series No. 912.

WHO, 2004. The World Health Report 2004 - Changing History. World Health Organization, Geneva.

Williams, E.H., Hayes, R.J., Smith, P.G., 1986. Admissions to a rural hospital in the West Nile district of Uganda over a 27 year period. Am. J. Trop. Med. Hyg. 89, 193-211. 